\title{
NONLINEAR STABILITY CONDITIONS AND A PRIORI ESTIMATES FOR BAROTROPIC HYDRODYNAMICS
}

\author{
Darryl D. HOLM a Jerrold E. MARSDEN b,1, Tudor RATIU c, ${ }^{2}$ and Alan WEINSTEIN b, 1 \\ ${ }^{a}$ Center for Nonlinear Studies and Theoretical Division, Los Alamos National Laboratory, Los Alamos, NM 87545, USA \\ ${ }^{\mathrm{b}}$ Department of Mathematics, University of California, Berkeley, CA $94720, U S A$ \\ ${ }^{c}$ Department of Mathematics, University of Arizona, Tucson, AZ 85721, USA
}

Received 29 June 1983

\begin{abstract}
A method developed by Arnold to prove nonlinear stability of certain steady states for ideal incompressible flow in two dimensions is extended to the barotropic compressible case. The results are applied to plane shear flow.
\end{abstract}

This paper uses a convexity method due to Arnold [1] to prove a priori stability estimates for smooth solutions of planar ideal barotropic fluid flows. The estimates obtained give $\mathrm{L}^{2}$ bounds on perturbations of momentum density, mass density and vorticity for a given stationary solution whose Bernoulli function and internal energy density satisfy certain inequalities. The estimates are global in time and are valid as long as the solutions being estimated remain smooth and, in a sense made precise, do not undergo cavitation. Arnold used this method to study the incompressible planar case, where his stability criteria established the nonlinear stability of flows satisfying Rayleigh's inflection point criterion. We develop similar results for the compressible case. We note that $\mathrm{L}^{2}$ estimates are obtained on the vorticity but not on the divergence of the velocity field. This is consistent with the formation of shocks, which are generally expected; at such times, the solutions cease to be smooth and our results cease to apply.

The idea for the method depends on the hamiltonian structure for compressible flow which has recently been developed by Morrison and Greene [2], Holm and Kupershmidt [3] and, in a way corresponding to Arnold's method for incompressible flow, by Marsden, Ratiu and Weinstein [4]. One finds that the equations are LiePoisson equations on the dual of the Lie algebra of the semi-direct product of vector fields and functions. On each coadjoint orbit (the "Lin constraint") the equations are hamiltonian in the classical sense, and critical points of the hamiltonian restricted to an orbit correspond to stationary solutions. Arnold [5,6] developed a formal stability criterion by demanding that the second variation of the hamiltonian restricted to the orbit be definite. We call this formal because in the infinite-dimensional case of concern to us, this method does not give a rigorous proof of nonlinear stability due to difficulties with the topologies involved. This was pointed out in Marsden and Abraham [7] and Ebin and Marsen [8] and must have been known to Arnold. There are similar difficulties with stability in elasticity and it is known that the second-variation method can, in a certain sense, fail $[9,10]$. The formal stability method gives stability for linearized solutions but does not, as formulated, prove nonlinear stability. This method has been used for example by Benzi et al. [11] to prove formal stability of certain planetary vortices.

Arnold [1] developed a convexity method which overcomes the difficulties with formal stability. The method is motivated by the formal stability arguments, but does not, strictly speaking, depend on them. The crucial

\footnotetext{
1 Partially supported by DOE contract AT03-82ER 12097.

2 Partially supported by NSF grant MCS 81-01642.
} 
point is to add to the hamiltonian $E$ a conserved functional $F$ which plays the role of a Lagrange multiplier constraint for the orbit. Then one uses convexity methods to estimate $E+F$ for finite perturbations of a given stationary solution.

It is interesting to note that formal stability criteria similar to Arnold's were developed for plasma theory by Kruskal and Oberman [12] and that a nonlinear stability method similar to Arnold's convexity method was developed by Newcomb [13] and by Rosenbluth [14].

Our results are limited to smooth compressible flows. It is known that $\omega / \rho=$ vorticity/density is not transported across shocks, and additional $\omega / \rho$ can be created by the shocks. It is not known how to deal with this problem.

In a forthcoming paper, we shall deal with additional examples: adiabatic three-dimensional compressible flow, two-dimensional magnetohydrodynamics, the Poisson-Vlasov equations for one-dimensional plasmas, and multilayer quasigeostrophic systems.

We shall denote stationary solutions with a subscript $\mathrm{s}$. Thus, $v_{\mathrm{s}}, \rho_{\mathrm{s}}$ and $\omega_{\mathrm{s}}$ are the velocity, density and vorticity of a stationary solution. The finite perturbations are denoted $\mathbf{v}=\mathbf{v}_{\mathrm{s}}+\Delta \mathbf{v}, \rho=\rho_{\mathrm{s}}+\Delta \rho$ and $\omega=\omega_{\mathrm{s}}+\Delta \omega$. Note that $\Delta$ denotes a finite perturbation and not the laplacian.

Consider compressible fluid flow taking place in a domain $D \subset R^{2}$ with smooth boundary. The barotropic fluid equations define a dynamical system in the space of fluid velocities $v(x, y, t)$ and mass densities $\rho(x, y, t)$ by

$\mathrm{d} \mathbf{v} / \mathrm{d} t=-\nabla h(\rho), \quad \mathrm{d} \rho / \mathrm{d} t=-\rho \operatorname{div} \mathbf{v}$,

where $\mathrm{d} / \mathrm{d} t=\partial / \partial t+\mathrm{v} \cdot \nabla$ is the material derivative and $h(\rho)$ is the specific enthalpy, a given function, related to the barotropic pressure, $p(\rho)$, by $\mathrm{d} h / \mathrm{d} \rho=\rho^{-1} \mathrm{~d} p / \mathrm{d} \rho$.

Planar fluid flow is thought of as taking place in the $(x, y)$ plane of $\mathrm{R}^{3}$. Denoting by $n$ the positively oriented unit vector along the $z$ axis, the vorticity $\omega$ is defined to be the real-valued function on $\mathrm{R}^{2}$ given by $\omega=n \cdot \operatorname{curl}$ $\boldsymbol{v}=v_{2 x}-v_{1 y}$, where $f_{x}=\partial f / \partial x$ and $f_{y}=\partial f / \partial y$ denote the partial derivatives of the real-valued function $f$. Using the relations $\nabla\left(v^{2} / 2\right)=(\mathbf{v} \cdot \nabla) \mathbf{v}-\omega n \times \mathbf{v}$ and $\operatorname{div}(\rho \mathbf{v})=(\mathbf{v} \cdot \nabla) \rho+\rho \operatorname{div}(\mathbf{v})$ in eqs. (1) and applying the operator $n \cdot$ curl to the first equation yields $\mathrm{d} \omega / \mathrm{d} t=-\omega \operatorname{div} v$, which combined with the second equation of (1) implies $\mathrm{d}(\omega / \rho) / \mathrm{d} t=0$, so that for every real-valued function of a real variable $\Phi(\xi)$, the functional

$F_{\Phi}(\omega / \rho):=\iint_{D} \rho \Phi(\omega / \rho) \mathrm{d} x \mathrm{~d} y$

is a conserved quantity of the system (1) (provided the integral exists and the solutions are smooth). Another conserved quantity is the total energy

$E:=\iint_{D}\left[\rho v^{2} / 2+\epsilon(\rho)\right] \mathrm{d} x \mathrm{~d} y$,

where $\epsilon(\rho)$ is the internal energy density of the fluid, related to the specific enthalpy by $\mathrm{d} \epsilon / \mathrm{d} \rho=h(\rho)$.

The equilibrium states of the system (1) are the stationary, two-dimensional, barotropic flows. For such stationary flows, the gradient vectors $\nabla\left(v_{\mathrm{s}}^{2} / 2+h\left(\rho_{\mathrm{s}}\right)\right)$ and $\nabla\left(\omega_{\mathrm{s}} / \rho_{\mathrm{s}}\right)$ can be shown to be orthogonal to the velocity. Consequently, these gradient vectors are collinear, provided they do not vanish. A sufficient condition for this collinearity is the functional relationship

$v_{\mathrm{s}}^{2} / 2+h\left(\rho_{\mathrm{s}}\right)=k\left(\omega_{\mathrm{s}} / \rho_{\mathrm{s}}\right)$

for some function $k(\xi), \xi \in \mathrm{R}$, defined wherever $\nabla\left(\omega_{\mathrm{s}} / \rho_{\mathrm{s}}\right)$ does not vanish; $k$ is called the Bernoulli function and relation (6) represents Bernoulli's law. Applying the operator $\left(\rho_{\mathrm{s}} / \omega_{\mathrm{s}}\right) n \times \nabla$ to (2) we get

$\rho_{\mathrm{s}} \mathbf{v}_{\mathrm{s}}=\left(\rho_{\mathrm{s}} / \omega_{\mathrm{s}}\right) n \times \nabla k\left(\omega_{\mathrm{s}} / \rho_{\mathrm{s}}\right)=\left(\rho_{\mathrm{s}} / \omega_{\mathrm{s}}\right)\left(-k_{y}\left(\omega_{\mathrm{s}} / \rho_{\mathrm{s}}\right), k_{x}\left(\omega_{\mathrm{s}} / \rho_{\mathrm{s}}\right)\right)$. 
We shall now prove the following proposition:

Proposition. Within the framework of smooth solutions with velocity fields parallel to the boundary and fixed circulation on the boundary, a stationary solution $v_{s}, \rho_{s}$ of the ideal compressible barotropic fluid equations (1) is a conditional extremum point of the total energy $E$ for fixed vorticity integral $F_{\Phi}$ and an absolute extremum point of $H=E+F_{\Phi}$, where

$\Phi(\zeta)=\zeta\left(\int^{\zeta} \frac{k(t)}{t^{2}} \mathrm{~d} t+\right.$ const $)$

$k$ being the function constant on streamlines given by Bernoulli's law.

After integration by parts, the derivative of $H$ at $(\mathbf{v}, \rho)$ in the direction $\Delta \mathbf{v}, \Delta \rho$ is

$$
\begin{aligned}
& \mathrm{D} H(\mathbf{v}, \rho) \cdot(\Delta \mathbf{v}, \Delta \rho)=\iint_{\mathrm{D}}\left\{\left[v^{2} / 2+h(\rho)+\Phi(\omega / \rho)-(\omega / \rho) \Phi^{\prime}(\omega / \rho) \Delta \rho\right.\right. \\
& \left.\quad+\left[\rho \mathbf{v}-\boldsymbol{n} \times \nabla \Phi^{\prime}(\omega / \rho)\right] \cdot \Delta \mathbf{v}\right\} \mathrm{d} x \mathrm{~d} y+\oint_{\partial \mathrm{D}} \Phi^{\prime}(\omega / \rho) \Delta \mathbf{v} \cdot \mathrm{d} l .
\end{aligned}
$$

For a stationary solution, the connected components of the boundary $\partial \mathrm{D}$ are streamlines. Since for stationary solutions, flow lines and streamlines coincide, and $\omega_{\mathrm{s}} / \rho_{\mathrm{s}}$ is constant along the flow, it follows that $\Phi^{\prime}\left(\omega_{\mathrm{s}} / \rho_{\mathrm{s}}\right)$ is constant on the components of $\partial \mathrm{D}$. Thus, the boundary term becomes

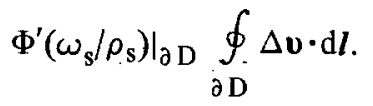

Since the variations satisfy $\oint \Delta v \cdot \mathrm{d} l=0$ for each connected component $C_{i}$ of $\partial \mathrm{D}$, the boundary term $\mathrm{C}_{i}$ vanishes. Eq. (4) shows that $\mathrm{DH}\left(\mathrm{v}_{\mathrm{s}}, \rho_{\mathrm{s}}\right)$ vanishes for a stationary flow obeying (2), provided that $\Phi$ is related to the Bernoulli function by $k(\zeta)+\Phi(\zeta)-\zeta \Phi^{\prime}(\zeta)=0$, from which the proposition follows. Differentiating with respect to $\zeta$ implies $\zeta^{-1} k^{\prime}(\zeta)-\Phi^{\prime \prime}(\zeta)=0$. The second term in the double integral of (4) vanishes since $\nabla \Phi^{\prime}\left(\omega_{\mathrm{s}} / \rho_{\mathrm{s}}\right)=\left(\rho_{\mathrm{s}} /\right.$ $\left.\omega_{\mathrm{s}}\right) \nabla k\left(\omega_{\mathrm{s}} / \rho_{\mathrm{s}}\right)$.

In a finite-dimensional hamiltonian system, it is a classical result of Lagrange that definiteness of the second derivative of the hamiltonian at a fixed point of the flow implies the Lyapunov stability of the fixed point [15]. In the infinite-dimensional case, there are difficulties with this idea due to the inconsistency between the function-space topology needed for the infinite-dimensional calculus to work and the topology of the second variation. (See, e.g. refs. [9,10] and ref. [4], Ch. 6 for relevant examples in elasticity.) However, it is instructive to carry out a formal stability argument for an infinite-dimensional system to see what kind of expressions one might hope to bound by use of a rigorous stability argument. Since the compressible ideal barotropic fluid equations are hamiltonian [2-4] such a formal analysis is applicable in this case.

We start with the remark that instead of the energy, one can use $H=E+F_{\Phi}$ as the hamiltonian. This is because $\Phi$ is such that the stationary flows are critical points of $H$ and $F_{\Phi}$ Poisson-commutes with any function since it is an orbit invariant $\left(F_{\Phi}\right.$ is, thus, a Casimir function in the sense of Weinstein [17]).

In the class of smooth solutions with fixed circulations on the boundary components, we have

$\mathrm{D} H(\mathbf{v}, \rho) \cdot(\Delta \mathbf{v}, \Delta \rho)=\iint\left\{\left[v^{2} / 2+h(\rho)-k(\omega / \rho)\right] \Delta \rho+[\rho \mathbf{v}-(\rho / \omega) n \times \nabla k(\omega / \rho)] \cdot \Delta \mathbf{v}\right\} \mathrm{d} x \mathrm{~d} y$

The quadratic form defined by the second derivative at the stationary solution is 


$$
\begin{aligned}
& \mathrm{D}^{2} H\left(\mathbf{v}_{\mathrm{s}}, \rho_{\mathrm{s}}\right) \cdot(\Delta \mathbf{v}, \Delta \rho)^{2}=\iint_{\mathrm{D}}\left\{\rho_{\mathrm{s}}\left|\Delta \mathbf{v}+\mathbf{v}_{\mathrm{s}} \Delta \rho / \rho_{\mathrm{s}}\right|^{2}+\left[\epsilon^{\prime \prime}\left(\rho_{\mathrm{s}}\right)-v_{\mathrm{s}}^{2} / \rho_{\mathrm{s}}\right](\Delta \rho)^{2}\right. \\
& \left.+\omega_{\mathrm{s}}^{-1} k^{\prime}\left(\omega_{\mathrm{s}} / \rho_{\mathrm{s}}\right)\left[\left(\rho_{\mathrm{s}} \Delta \omega-\omega_{\mathrm{s}} \Delta \rho\right) / \rho_{\mathrm{s}}\right]^{2}\right\} \mathrm{d} x \mathrm{~d} y .
\end{aligned}
$$

This form is positive definite if and only if:

(i) $\epsilon^{\prime \prime}\left(\rho_{\mathrm{s}}\right)-v_{\mathrm{s}}^{2} / \rho_{\mathrm{s}}=\left(c_{\mathrm{s}}^{2}-v_{\mathrm{s}}^{2}\right) / \rho_{\mathrm{s}}>0$,

where $c_{\mathrm{s}}$ is the sound speed of the stationary solution defined by $\rho_{\mathrm{s}} \epsilon^{\prime \prime}\left(\rho_{\mathrm{s}}\right)=c_{\mathrm{s}}^{2}$ is the sound speed of the stationary solution defined by $\rho_{\mathrm{s}} \epsilon^{\prime \prime}\left(\rho_{\mathrm{s}}\right)=c_{\mathrm{s}}^{2}$, i.e., the stationary flow is everywhere subsonic, and

(ii) $\left(\rho_{\mathrm{s}} / \omega_{\mathrm{s}}\right) k^{\prime}\left(\omega_{\mathrm{s}} / \rho_{\mathrm{s}}\right)=\Phi^{\prime \prime}\left(\omega_{\mathrm{s}} / \rho_{\mathrm{s}}\right)=\nabla\left(\mathrm{v}_{\mathrm{s}}^{2} / 2+h\left(\rho_{\mathrm{s}}\right)\right) / \nabla\left(\frac{1}{2}\left(\omega_{\mathrm{s}} / \rho_{\mathrm{s}}\right)^{2}\right)>0$

(recall that these two gradients are collinear), i.e., these two gradient vectors point in the same direction throughout the stationary flow.

Formally stable flows are also possible when the expression (5) is negative definite. This can happen only if the flow is supersonic enough that the middle term overcomes the first and third terms. For $\rho_{\mathrm{s}}=1$, and $\Delta \rho=0$, formula (5) reduces to Arnold's result [5]..

We shall now make a modified stability argument rigorous in the context of smooth solutions, and shall address the question of non-linear (Lyapunov) stability. This will be done by finding a priori estimates on the perturbations which will be expressed only in terms of the Bernoulli function $k$. We hasten to add that our analysis holds only for the smooth regime; for if shocks occur, and this is expected to happen generically, the quantity $\omega / \rho$ need not be conserved along the flow lines (cf. ref. [18] pp. 221, 222).

Theorem 1. Assume that the Bernoulli function $k$ and the internal energy density satisfy

$0<a \leqslant \zeta^{-1} k^{\prime}(\zeta) \leqslant A<\infty$,

where $a$ and $A$ are positive constants and similarly,

$0<e_{1} \leqslant \epsilon^{\prime \prime}(\tau) \leqslant e_{2}<\infty$,

with constants $e_{1}, e_{2}$, and for all values of the arguments. Let $(\Delta v, \Delta \rho)$ be a smooth perturbation of a stationary solution $\left(v_{\mathrm{s}}, \rho_{\mathrm{s}}\right)$ and denote its value at $t=0$ by $\left(\Delta \mathbf{v}_{0}, \Delta \rho_{0}\right)$. Assume that the circulation of $\Delta \mathbf{v}_{0}$ on each component of $\partial \mathrm{D}$ is zero. Then the perturbation $(\Delta \mathbf{v}, \Delta \rho)$ of the stationary solution $\left(\mathbf{v}_{\mathrm{s}}, \rho_{\mathrm{s}}\right)$ at any time $t$ is estimated in terms of $\left(\Delta v_{0}, \Delta \rho_{0}\right)$ by

$$
\begin{aligned}
& \iint_{\mathrm{D}}\left[\frac{\left.\Delta(\rho \mathbf{v})\right|^{2}}{\rho_{\mathrm{s}}+\Delta \rho}+\left(e_{1}-\frac{v_{\mathrm{s}}^{2}}{\rho_{\mathrm{s}}+\Delta \rho}\right)(\Delta \rho)^{2}+a\left(\rho_{\mathrm{s}}+\Delta \rho\right)[\Delta(\omega / \rho)]^{2}\right] \mathrm{d} x \mathrm{~d} y \\
& \quad \leqslant \int_{\mathrm{D}}\left[\frac{\left|\Delta(\rho \mathbf{v})_{0}\right|^{2}}{\rho_{\mathrm{s}}+\Delta \rho_{0}}+\left(e_{2}-\frac{v_{\mathrm{s}}^{2}}{\rho_{\mathrm{s}}+\Delta \rho_{0}}\right)\left(\Delta \rho_{0}\right)^{2}+A\left(\rho_{\mathrm{s}}+\Delta \rho_{0}\right)\left[\Delta(\omega / \rho)_{0}\right]^{2}\right] \mathrm{d} x \mathrm{~d} y .
\end{aligned}
$$

Proof. Since the circulation of $\Delta \mathbf{v}_{0}$ is zero initially on the components of $\partial \mathrm{D}$, the circulation of $\Delta \mathbf{v}$ at any later time will also be zero on the components of $\partial \mathrm{D}$, by Kelvin's circulation theorem. Since $E$ and $F_{\Phi}$ are conserved along the flow of (1), so is $H\left(v_{\mathrm{s}}+\Delta \mathrm{v}, \rho_{\mathrm{s}}+\Delta \rho\right)-H\left(v_{\mathrm{s}}, \rho_{\mathrm{s}}\right)$. With the choice of $\Phi$ of our proposition, $\mathrm{D} H\left(\mathbf{v}_{\mathrm{s}}, \rho_{\mathrm{s}}\right)=0$ so that $\hat{H}(\Delta \mathbf{v}, \Delta \rho):=H\left(\mathbf{v}_{\mathrm{s}}+\Delta \mathbf{v}, \rho_{\mathrm{s}}+\Delta \rho\right)-H\left(\mathbf{v}_{\mathrm{s}}, \rho_{\mathrm{s}}\right)-\mathrm{D} H\left(\mathbf{v}_{\mathrm{s}}, \rho_{\mathrm{s}}\right) \cdot(\Delta \mathbf{v}, \Delta \rho)$ is also conserved. We shall estimate each summand of $H$. We have, after a short computation, 


$$
\begin{aligned}
& \hat{E}(\Delta \mathbf{v}, \Delta \rho):=E\left(\mathbf{v}_{\mathrm{s}}+\Delta \mathbf{v}, \rho_{\mathrm{s}}+\Delta \rho\right)-E\left(\mathbf{v}_{\mathrm{s}}, \rho_{\mathrm{s}}\right)-\mathrm{D} E\left(\boldsymbol{v}_{\mathrm{s}}, \rho_{\mathrm{s}}\right) \cdot(\Delta \mathbf{v}, \Delta \rho) \\
& =\iint_{D}\left\{\left[\frac{1}{2} \rho_{\mathrm{s}}|\Delta \mathbf{v}|^{2}+\left(\mathbf{v}_{\mathrm{s}} \cdot \Delta \mathbf{v}\right) \Delta \rho+\frac{1}{2} \Delta \rho|\Delta \mathbf{v}|^{2}\right]+\epsilon\left(\rho_{\mathrm{s}}+\Delta \rho\right)-\epsilon\left(\rho_{\mathrm{s}}\right)-\epsilon^{\prime}\left(\rho_{\mathrm{s}}\right) \Delta \rho\right\} \mathrm{d} x \mathrm{~d} y \\
& =\iint_{\mathrm{D}}\left(\frac{|\Delta(\rho \mathrm{v})|^{2}}{2\left(\rho_{\mathrm{s}}+\Delta \rho\right)}-\frac{1}{2} v_{\mathrm{s}}^{2} \frac{(\Delta \rho)^{2}}{\underline{\rho_{\mathrm{s}}+\Delta \rho}}+\left[\epsilon\left(\rho_{\mathrm{s}}+\Delta \rho\right)-\epsilon\left(\rho_{\mathrm{s}}\right)-\epsilon^{\prime}\left(\rho_{\mathrm{s}}\right) \Delta \rho\right]\right) \mathrm{d} x \mathrm{~d} y .
\end{aligned}
$$

According to the convexity hypotheses on $\epsilon$, we have the following inequalities, for all $\Delta \rho$ :

$e_{1}(\Delta \rho)^{2} / 2 \leqslant \epsilon\left(\rho_{\mathrm{s}}+\Delta \rho\right)-\epsilon\left(\rho_{\mathrm{s}}\right)-\epsilon^{\prime}\left(\rho_{\mathrm{s}}\right) \Delta \rho \leqslant e_{2}(\Delta \rho)^{2} / 2$.

Consequently, $\hat{E}$ is bounded from above and below by

$$
\begin{aligned}
& 2 \hat{E}(\Delta \mathbf{v}, \Delta \rho) \geqslant \iint_{\mathrm{D}}\left[\frac{|\Delta(\rho \mathbf{v})|^{2}}{\rho_{\mathrm{s}}+\Delta \rho}+\left(e_{1}-\frac{v_{\mathrm{s}}^{2}}{\rho_{\mathrm{s}}+\Delta \rho}\right)(\Delta \rho)^{2}\right] \mathrm{d} x \mathrm{~d} y, \\
& 2 \hat{E}(\Delta \mathbf{v}, \Delta \rho) \leqslant \iint_{\mathrm{D}}\left[\frac{|\Delta(\rho \mathbf{v})|^{2}}{\rho_{\mathrm{s}}+\Delta \rho}+\left(e_{2}-\frac{v_{\mathrm{s}}^{2}}{\rho_{\mathrm{s}}+\Delta \rho}\right)(\Delta \rho)^{2}\right] \mathrm{d} x \mathrm{~d} y .
\end{aligned}
$$

Next, we need to estimate the functional

$\hat{F}_{\Phi}(\Delta \mathbf{v}, \Delta \rho)=F_{\Phi}\left(\mathbf{v}_{\mathrm{s}}+\Delta \mathbf{v}, \rho_{\mathrm{s}}+\Delta \rho\right)-F_{\Phi}\left(\mathbf{v}_{\mathrm{s}}, \rho_{\mathrm{s}}\right)-\mathrm{D} F_{\Phi}\left(\mathbf{v}_{\mathrm{s}}, \rho_{\mathrm{s}}\right) \cdot(\Delta \mathbf{v}, \Delta \rho)$

for $\Phi$ given by our proposition. We have

$$
\begin{gathered}
\hat{F}_{\Phi}(\Delta \boldsymbol{v}, \Delta \rho)=\iint_{\mathrm{D}}\left[\left(\rho_{\mathrm{s}}+\Delta \rho\right) \Phi\left(\frac{\omega_{\mathrm{s}}+\Delta \omega}{\rho_{\overline{\mathrm{s}}}+\Delta \boldsymbol{\Delta}}\right)-\rho_{\mathrm{s}} \Phi\left(\omega_{\mathrm{s}} / \rho_{\mathrm{s}}\right)-\left[\Phi\left(\omega_{\mathrm{s}} / \rho_{\mathrm{s}}\right)-\left(\omega_{\mathrm{s}} / \rho_{\mathrm{s}}\right) \Phi^{\prime}\left(\omega_{\mathrm{s}} / \rho_{\mathrm{s}}\right)\right] \Delta \rho\right. \\
\left.-\Phi^{\prime}\left(\omega_{\mathrm{s}} / \rho_{\mathrm{s}}\right) \Delta \omega\right] \mathrm{d} x \mathrm{~d} y=\iint_{\mathrm{D}}\left(\rho_{\mathrm{s}}+\Delta \rho\right)\left[\Phi\left(\frac{\omega_{\mathrm{s}}+\Delta \omega}{\rho_{\mathrm{s}}+\Delta \rho}\right)-\Phi\left(\omega_{\mathrm{s}} / \rho_{\mathrm{s}}\right)-\Phi^{\prime}\left(\omega_{\mathrm{s}} / \rho_{\mathrm{s}}\right) \Delta(\omega / \rho)\right] \mathrm{d} x \mathrm{~d} y,
\end{gathered}
$$

because

$\Delta(\omega / \rho)=\left(\omega_{\mathrm{s}}+\Delta \omega\right) /\left(\rho_{\mathrm{s}}+\Delta \rho\right)-\omega_{\mathrm{s}} / \rho_{\mathrm{s}}=\left(\rho_{\mathrm{s}} \Delta \omega-\omega_{\mathrm{s}} \Delta \rho\right) / \rho_{\mathrm{s}}\left(\rho_{\mathrm{s}}+\Delta \rho\right)$.

From (6), convexity of $\Phi(\zeta)$ implies

$\frac{1}{2} \mathrm{a}[\Delta(\omega / \rho)]^{2} \leqslant \Phi\left(\omega_{\mathrm{s}} / \rho_{\mathrm{s}}+\Delta(\omega / \rho)\right)-\Phi\left(\omega_{\mathrm{s}} / \rho_{\mathrm{s}}\right)-\Phi^{\prime}\left(\omega_{\mathrm{s}} / \rho_{\mathrm{s}}\right) \Delta(\omega / \rho) \leqslant \frac{1}{2} A[\Delta(\omega / \rho)]^{2}$, since $\zeta^{-1} k^{\prime}(\zeta)=\Phi^{\prime \prime}(\zeta)$. Consequently, $\hat{F}_{\Phi}$ is bounded by

$a \iint_{D}\left(\rho_{\mathrm{s}}+\Delta \rho\right)[\Delta(\omega / \rho)]^{2} \mathrm{~d} x \mathrm{~d} y \leqslant 2 \hat{F}_{\Phi}(\Delta \mathbf{v}, \Delta \rho) \leqslant A \iint_{\mathrm{D}}\left(\rho_{\mathrm{s}}+\Delta \rho\right)[\Delta(\omega / \rho)]^{2} \mathrm{~d} x \mathrm{~d} y$

By (10) and (11) we find that $2 \hat{H}(\Delta \mathbf{v}, \Delta \rho)\left[=2 \hat{H}\left(\Delta \mathbf{v}_{0}, \Delta \rho_{0}\right)\right]$ is bounded from below (above) by the left (right) hand side of $(8)$, which proves the theorem.

The a priori estimate in the theorem implies stability for smooth solutions, provided $\rho_{\mathrm{s}}+\Delta \rho$ remains bounded away from zero. Under these hypotheses, for $e_{1}$ big enough, the left-hand side of the inequality can serve as a measure of "smallness" with respect to which solutions starting "near" the stationary solution remain "close" to it. Our hypothesis requiring $\rho=\rho_{\mathbf{s}}+\Delta \rho$ to stay bounded away from zero excludes specifically the phenomenon 
of cavitation. In any case, the inequality in the theorem shows that, up to the first shock time, the perturbed solution cannot stray too far away from the stationary solution in the $\mathrm{L}^{2}$ type norm of the perturbed momentum density $\Delta(\rho v)$, the perturbed density, and perturbed "vorticity" $\Delta(\omega / \rho)$ given by the left-hand side of $(8)$. Note that we do not have an $\mathrm{L}^{2}$ bound on the divergence of the momentum density. This is to be expected, since such a bound does not even occur in the linearized situation [see eq. (5)].

An expedient way to exclude cavitation is to strengthen hypothesis (7) in the theorem by replacing it with

$0<e_{1} \rho_{\mathrm{s}}(\Delta \rho)^{2} / 2\left(\rho_{\mathrm{s}}+\Delta \rho\right) \leqslant \epsilon\left(\rho_{\mathrm{s}}+\Delta \rho\right)-\epsilon\left(\rho_{\mathrm{s}}\right)-\epsilon^{\prime}\left(\rho_{\mathrm{s}}\right) \Delta \rho \leqslant e_{2} \rho_{\mathrm{s}}(\Delta \rho)^{2} / 2\left(\rho_{\mathrm{s}}+\Delta \rho\right)<\infty$.

In this case of an "elastic fluid" (so-called, since such inequalities appear in elasticity, indicating that it would take an infinite amount of energy to tear the fluid apart) the a priori estimate becomes (8), with $e_{1}$ and $e_{2}$ replaced by $e_{1} \rho_{\mathrm{s}} /\left(\rho_{\mathrm{s}}+\Delta \rho\right)$ and $e_{2} \rho_{\mathrm{s}} /\left(\rho_{\mathrm{s}}+\Delta \rho_{0}\right)$.

The bound (12) is satisfied by an equation of state of Mie-Grüneisen type [19]. Namely, $\epsilon(\rho)=A / \rho+B \rho+C$; the constants $A, B, C$, are $A=e \rho_{\mathrm{s}}^{3} / 2, B=\epsilon^{\prime}\left(\rho_{\mathrm{s}}\right)+e \rho_{\mathrm{s}} / 2, C=\epsilon\left(\rho_{\mathrm{s}}\right)-\rho_{\mathrm{s}} \epsilon^{\prime}\left(\rho_{\mathrm{s}}\right)-e \rho_{\mathrm{s}}^{2}$, and $e_{1} \leqslant e \leqslant e_{2}$.

When inequalities (6) are reversed and estimates are made of the concavity of $F_{\Phi}$, one can treat the case where $\Phi^{\prime \prime}<0$.

Theorem 2. Under the same hypotheses as in Theorem 1 but with (6) replaced by

$0<a \leqslant-\zeta^{-1} k^{\prime}(\zeta) \leqslant A<\infty$,

an estimate of the perturbation $(\Delta v, \Delta \rho)$ of the stationary solution $\left(\boldsymbol{v}_{\mathrm{s}}, \rho_{\mathrm{s}}\right)$ (with the circulation of $\Delta \mathbf{v}_{0}$ vanishing on each connected component of $\partial \mathrm{D})$ is given in terms of the initial perturbation $\left(\Delta \mathbf{v}_{0}, \Delta \rho_{0}\right)$ by

$$
\begin{aligned}
& \iint_{\mathrm{D}}\left[a\left(\rho_{\mathrm{s}}+\Delta \rho\right)[\Delta(\omega / \rho)]^{2}-\frac{|\Delta(\rho \mathbf{v})|^{2}}{\rho_{\mathrm{s}}+\Delta \rho}-\left(e_{2}-\frac{v_{\mathrm{s}}^{2}}{\rho_{\mathrm{s}}+\Delta \rho}\right)(\Delta \rho)^{2}\right] \mathrm{d} x \mathrm{~d} y \\
& \quad \leqslant \iint_{\mathrm{D}}\left[A\left(\rho_{\mathrm{s}}+\Delta \rho_{0}\right)\left[\Delta(\omega / \rho)_{0}\right]^{2}-\frac{\left|\Delta(\rho \mathbf{v})_{0}\right|^{2}}{\rho_{\mathrm{s}}+\Delta \rho_{0}}-\left(e_{1}-\frac{v_{\mathrm{s}}^{2}}{\rho_{\mathrm{s}}+\Delta \rho_{0}}\right)\left(\Delta \rho_{0}\right)^{2}\right] \mathrm{d} x \mathrm{~d} y .
\end{aligned}
$$

The proof is similar to that of theorem 1 , except working with $-\hat{H}$, and the same comments hold regarding cavitation. However, in order to get a stability estimate, one must now require that the left hand side of (13) be positive definite. This could conceivably happen for a sufficiently supersonic flow.

Example: Shear Flow. A stationary solution of (1) in the strip $\left\{(x, y) \in \mathrm{R}^{2} \mid Y_{1} \leqslant y \leqslant Y_{2}\right\}$, is given by the plane parallel flows with arbitrary velocity profile $v_{s}(x, y)=(u(y), 0)$ and constant density $\rho_{\mathrm{s}}=1$. We can allow $x$ to be unrestricted in $\mathbf{R}$ or to be periodic. In the former case, we require that the perturbations allowed be initially square integrable in the sense that the right-hand side of $(13)$ be finite. Note that $\left(\omega_{s} / \rho_{s}\right)(x, y)=-u^{\prime}(y)$. Let $c_{\mathrm{s}}$ denote the sound speed of this stationary solution. By our earlier analysis this flow is formally, hence linearized, stable if and only if $c_{\mathrm{s}}^{2}-u(y)^{2}>0$ and $u(y) / u^{\prime \prime}(y)>0$. By exploiting the translational symmetry of the problem, one finds that linearized stability also holds if $\left[u(y)-u_{0}\right] / u^{\prime \prime}(y)>0$ for a constant $u_{0}$. The hypothesis on the existence of the Bernoulli function $k$ is in this case $u^{\prime \prime}(y) \neq 0$. In other words, plane parallel flows with constant density and velocity profile with no inflection point are formally, hence linearly, stable. The analogous result in the incompressible problem is called Rayleigh's theorem [20]. Subsequent developments in the incompressible case are discussed, e.g., by Drazin and Reid [21].

We turn now to the study of our a priori estimates for this shear flow. For this, we must compute the Bernoulli function $k$ from its defining relation (2) under the hypothesis $\nabla\left(\omega_{s} / \rho_{\mathrm{s}}\right)=-u^{\prime \prime}(y) \neq 0$. Denoting by $\phi$ the inverse of $u$, we get $k(\zeta)=u[\phi(\zeta)]^{2} / 2+h(1)$ and thus $\left.k^{\prime}(\zeta)=-u(\phi(\zeta)) u^{\prime}(\phi(\zeta)) / u^{\prime \prime}(\zeta)\right)=\zeta u(\phi(\zeta)) / u^{\prime \prime}(\phi(\zeta))$, so that condition

(6) of theorem 1 becomes 
$0<a \leqslant u(y) / u^{\prime \prime}(y) \leqslant A<\infty$.

To get the a priori estimate (8), one imposes condition (7), which bounds $\epsilon^{\prime \prime}(\tau)$. Condition (7), for example, is satisfied for an ideal gas with $\gamma=2$, i.e., a monatomic gas in two dimensions. The a priori estimate (8) then results, with $\rho_{\mathrm{s}}=1$ and velocity profile, $u(y)$, satisfying (14), but arbitrary otherwise.

For the Mie--Grüneisen equation of state condition (14) is sufficient for the a priori estimate for the "elastic fluid", again with $\rho_{\mathrm{s}}=1$.

Parallel shear flows with one inflection point taking place at $y=0\left[u^{\prime \prime}(0)=0\right]$ can also be considered, under the assumption that the velocity profile is antisymmetric about the inflection point, $u(-y)=-u(y)$. For the case in which the ratio $u(y) / u^{\prime \prime}(y)$ is positive and bounded as in (14), one again obtains a priori bounds. For example, one may take $u(y)=\operatorname{arectanh} y$.

Compressible shear flow in the plane can also be stationary if $v_{s}(x, y)=(u(y), 0)$ and $\rho_{s}(x, y)=f(y)$, for arbitrary functions $u(y), f(y)$. In this case, $\omega_{\mathrm{s}}(x, y) / \rho_{\mathrm{s}}=-u^{\prime}(y) / f(y)$ and the assumption on the existence of the Bernoulli $k$ is $\left[u^{\prime}(y) / f(y)\right]^{\prime} \neq 0$. This flow is formally stable provided $c_{s}^{2}(y)-u^{2}(y)>0$ and $\zeta^{-1} k^{\prime}(\zeta)>0$, where $c_{\mathrm{s}}(y)$ is the sound speed. Thus, the stationary flow must be subsonic everywhere, and $k(\zeta)$ must be increasing as a function of $\zeta^{2} / 2$. The a priori estimate (8) holds, if $\epsilon$ and $k$ satisfy (6) and (7), respectively.

We are grateful to Ron DiPerra, Tom Beale, Andy Majda, D. ter Haar, and Steve Wan for their helpful comments on various aspects of this work.

\section{References}

[1] V.I. Arnold, Am. Math. Soc. Transl. 79 (1969) 267.

[2] P.J. Morrison and J.M. Greene. Phys. Rev. Lett. 45 (1980) 790.

[3] D. Holm and B. Kupershmidt, Physica 6D (1983) 347.

[4] J.E. Marsden, T. Ratiu and A. Weinstein, Semidirect products and reduction in mechanics, Trans. Amer. Math. Soc. to be published.

[5] V.I. Arnold, Sov. Math. Dokl. 162 (1965) 773.

[6] V.I. Arnold, Ann. Inst. Fourier (Grenoble) 16, (1966) 319.

[7] J.E. Marsden and R. Abraham, in: Proc. Symp. Pure Math., Vol. 16 (Am. Math. Soc., Providence, RI, 1970) pp. 237-243.

[8] D.G. Ebin and J. Marsden, Ann. Math. 92 (1970) 102.

[9] J.M. Ball, R.J. Knops and J.E. Marsden, Springer Lecture Notes in Math., Vol. 466 (Springer, Berlin, 1978) pp. 41-49.

[10] J. Ball and J. Marsden, Quasiconvexity, second variations and stability in nonlinear elasticity (1983), in preparation.

[11] R. Benzi, S. Pierini, A. Vulpiani and E. Salusti, Geophys. Astrophys. Fluid Dynamics 20 (1982) 293.

[12] M. Kruskal and C. Oberman, Phys. Fluids 1 (1958) No. 4.

[13] I.B. Bernstein, Phys. Rev. 109 (1958), 10, Appendix I.

[14] M.N. Rosenbluth, Advanced plasma theory (Academic Press, New York, 1964) p.137.

[15] V.I. Arnold, Mathematical methods of classical mechanics (Springer Berlin, 1978).

[16] J.E. Marsden and T.J.R. Hughes, Mathematical foundations of elasticity (Prentice-Hall, New York, 1983).

[17] A. Weinstein, The local structure of Poisson manifolds, J. Diff. Geom., to be published.

[18] J. Serrin, Handbuch der Physik, Vol. VIII/1 (Springer, Berlin, 1959) pp. 125-263.

[19] J.C. Slater, Introduction to chemical physics (McGraw-Hill, New York, 1939) ch. 13.

[20] Lord Rayleigh, Proc. London Math. Soc. 10 (1880) 4; Scientific Papers, Vol. 1 (Cambridge Univ. Press, London).

[21] P.G. Drazin and W.H. Reid, Hydrodynamic stability (Cambridge Univ. Press, London, 1981). 